\title{
Clinical efficacy and safety of fluoroquinolone containing regimens in patients with Mycobacterium avium complex pulmonary disease
}

\author{
To the Editor:
}

Mycobacterium avium complex pulmonary disease (MAC-PD) is an increasing problem worldwide [1]. The American Thoracic Society/Infectious Diseases Society of America (ATS/IDSA) guidelines recommend a macrolide-ethambutol-rifamycin combination as first-line treatment for MAC-PD (standard triple therapy) [2]. This therapy results in microbiological success in only $52-60 \%$ of patients $[3,4]$. Treatment discontinuation or modification due to toxicity is common $[5,6]$. Fluoroquinolones are frequently prescribed for MAC-PD [7]. Although favourable activity has been shown in vitro and in mouse models [8], there is little clinical evidence supporting their efficacy and safety for MAC-PD [9-11]. We sought to investigate treatment outcomes and adverse events among MAC-PD patients treated with fluoroquinolone-containing therapy versus standard triple therapy.

All patients seen in our clinic in Toronto, ON, Canada, from July 2003 to December 2016 were retrospectively reviewed. Patients were included if they were $\geqslant 18$ years old, met ATS/IDSA criteria for MAC-PD, and received $\geqslant 6$ months of treatment with three oral antibiotics. Patients with HIV infection, previous treatment for MAC-PD elsewhere without data available on treatment/outcomes, or aminoglycoside use in the first 6 months of treatment were excluded. Our institutional ethics board approved this study.

We defined a patient as having "received" a drug if it was prescribed for $\geqslant 2$ months. Patients were divided into two groups; those receiving standard triple therapy (macrolide-rifamycin-ethambutol), and those receiving fluoroquinolone-containing therapy, that is, $\geqslant 2$ months of a fluoroquinolone (ciprofloxacin, moxifloxacin, or levofloxacin), started within the first 6 months of treatment, plus macrolide and either ethambutol or rifamycin. Patients who had an additional antibiotic added to their regimen after the first 6 months of treatment were included in the analyses of treatment outcomes only until the fourth antibiotic was added. Chi-square tests were used to compare categorical variables and t-tests or Mann-Whitney tests were used to compare continuous variables. Two-sided p-values $<0.05$ were considered statistically significant.

During the study period, 300 MAC-PD patients were seen in our clinic and 103 patients met inclusion criteria; 61 were treated with standard triple therapy and 42 were treated with fluoroquinolone-containing therapy. Specific antibiotics are described in the table 1. Daily therapy (versus intermittent) was prescribed to $64 \%$ of patients in each group. Indications for fluoroquinolones included adverse events to rifamycin (9/ $42,21 \%)$, avoidance of rifamycin due to liver disease or drug interactions (14/42,33\%), adverse events to ethambutol $(5 / 42,12 \%)$, previous treatment with fluoroquinolones with acceptable response and tolerance (2/42, 5\%), concomitant suppressive therapy for Pseudomonas aeruginosa (7/42, 17\%), and unclear (5/42, 12\%).

Patients in the fluoroquinolone-containing therapy group were significantly older (table 1). Most patients had M. avium and nodular-bronchiectatic disease and the treatment duration did not differ between the two groups.

@ERSpublications

Fluoroquinolone containing regimens for Mycobacterium avium complex pulmonary disease demonstrated similar treatment outcomes, but more adverse events, than standard triple therapy http://bit.ly/2RdlFpa

Cite this article as: Khadawardi $\mathrm{H}$, Marras TK, Mehrabi M, et al. Clinical efficacy and safety of fluoroquinolone containing regimens in patients with Mycobacterium avium complex pulmonary disease. Eur Respir J 2020; 55: 1901240 [https://doi.org/10.1183/13993003.01240-2019]. 
TABLE 1 Baseline characteristics, treatment outcomes, and adverse events in patients with Mycobacterium avium complex (MAC) pulmonary disease treated with standard therapy versus fluoroquinolone-containing therapy

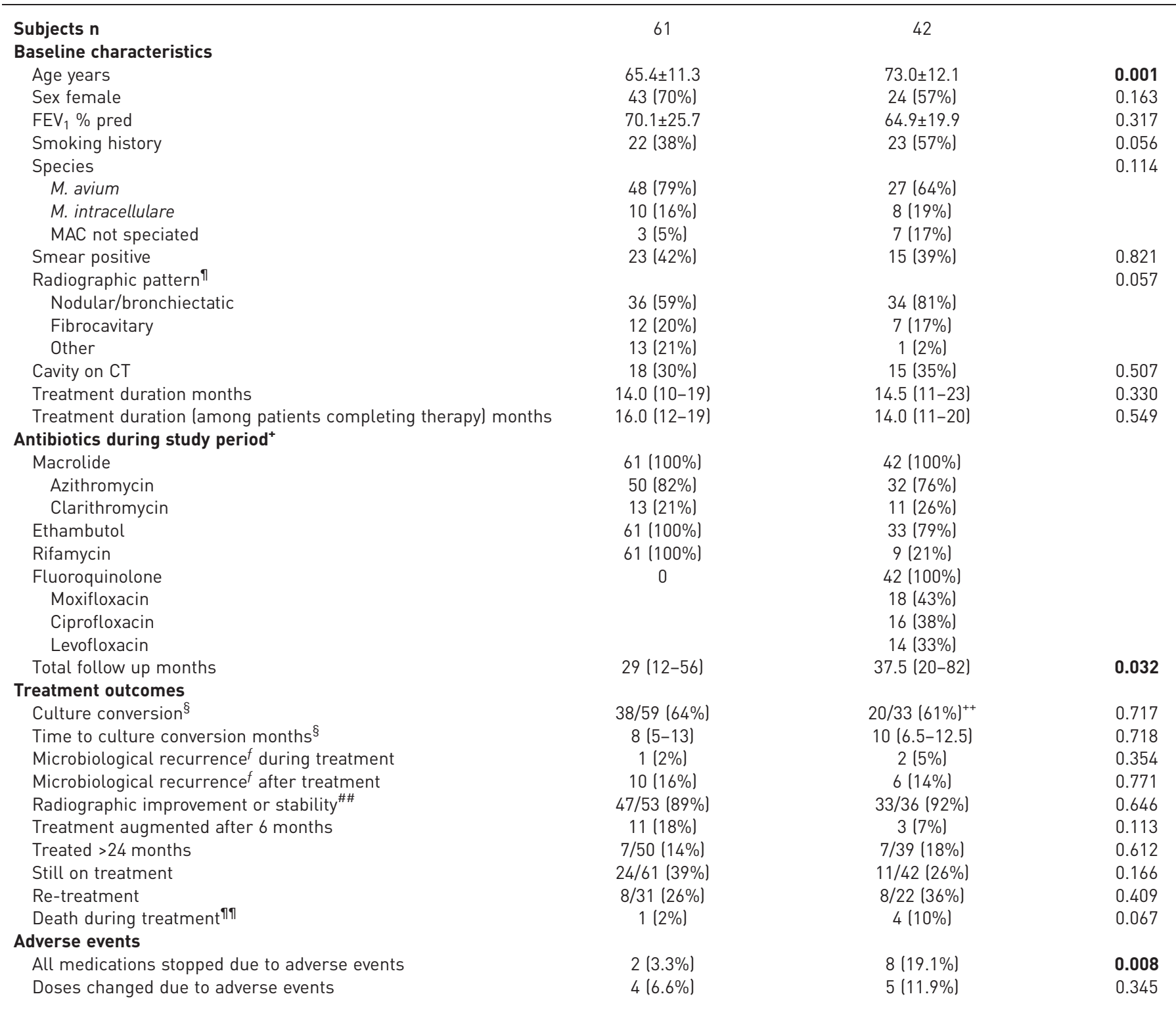

Data are presented as mean \pm SD or median (interquartile range), unless otherwise stated. FEV1: forced expiratory volume in $1 \mathrm{~s}$; CT: computed tomography. ": macrolide, ethambutol, and rifamycin. "ः CT pattern determined by the treating physician. ${ }^{+}$: medication prescribed for $\geqslant 2$ months. Values in each antibiotic class may not sum to $100 \%$ because some patients received $\geqslant 2$ months of more than 1 antibiotic in the class. ${ }^{\S}$ : three consecutive negative sputum cultures, or the inability to expectorate sputum on treatment, with time to conversion calculated from treatment initiation until the first negative culture. ${ }^{f}$ : microbiological recurrence defined as two positive sputum cultures after culture conversion. \#\#: based upon radiologist's report on CT scan closest to 6 months after treatment initiation. "ा?: causes of death were as follows: standard therapy group: 1) unknown in patient with chronic lymphocytic leukaemia; fluoroquinolone-containing therapy group: 1) unknown in patient with severe COPD, 2) respiratory failure in patient with severe cavitary MAC pulmonary disease, 3) cardiac arrest of unknown cause, and 4) unknown. ${ }^{++}: 11 / 42$ of these patients had drug susceptibility testing performed at some point before, during, or after treatment, and there was no association between moxifloxacin minimum inhibitory concentration and sputum culture conversion.

Among standard therapy group patients, 38/59 (64\%) achieved sputum culture conversion at a median (interquartile range) treatment duration of 8 (5-13) months, compared with 20/33 (60\%) fluoroquinolone-group patients at a median duration of $10(6.5-12.5)$ months $(p=0.817)$. There was no significant difference between groups in other examined outcomes (table 1). However, more patients in the fluoroquinolone-containing (versus standard) therapy group experienced adverse events leading to 
treatment discontinuation $(8 / 42(19 \%)$ versus $2 / 61$ (3\%) respectively, $\mathrm{p}=0.008)$, with no difference by specific fluoroquinolone $(\mathrm{p}=0.254)$.

To better characterise adverse events, we examined whether these patients ever received any of these antibiotics for their MAC-PD, at any time during follow-up (including $<2$ months or during re-treatment). All 103 patients received ethambutol for some duration, and among them 13 patients (13\%) stopped this medication due to an adverse event. No patients permanently discontinued a macrolide, but 4/103 (4\%) switched to a different drug in the class. Among patients who received a rifamycin, 10/83 (12\%) discontinued it due to an adverse event, and none switched within the class. Among 50 patients who received fluoroquinolones at any time during follow-up, seven (14\%) permanently discontinued the drug due to an adverse event, and $10(20 \%)$ switched within the class. Among these 50 patients, only one discontinued a fluoroquinolone due to palpitations, while $17 / 50$ had an electrocardiogram $\geqslant 2$ weeks after starting a fluoroquinolone, and none had a QTc $>500 \mathrm{~ms}$.

In our retrospective comparison of MAC-PD patients receiving fluoroquinolones substituted for a rifamycin or ethambutol versus standard triple therapy, we did not observe significant differences in outcomes, but more patients receiving fluoroquinolone-containing therapy stopped all treatment due to adverse events.

Patients in the fluoroquinolone-containing therapy group were significantly older and many received a fluoroquinolone because of a history of an adverse event to a rifamycin or ethambutol, or to avoid a rifamycin due to drug interactions or liver disease. Such patients may have more comorbidities and be more prone to adverse events, contributing to the higher rate of adverse events prompting treatment discontinuation, and the numerically higher number of deaths. When we looked at all patients who received these drugs for any duration, we found that $14 \%$ of patients who received a fluoroquinolone discontinued the class due to an adverse event, a similar proportion to ethambutol (13\%) and rifamycins (12\%). Electrocardiograms were performed in a minority of patients receiving fluoroquinolones; however, we did not observe significant QT prolongation.

Several prior studies evaluated fluoroquinolones for MAC-PD. In a prospective study, 13 patients who received gatifloxacin-rifampin-ethambutol achieved similar outcomes compared to 14 patients who received standard triple therapy [10]. Кон et al. [11] reported that the addition of moxifloxacin to standard triple therapy resulted in sputum culture conversion in 12/41 patients who had refractory MAC-PD. JENkINs et al. [9] performed a randomised trial involving 170 MAC-PD patients treated for 2 years; there was no significant difference in treatment outcomes between the group receiving ciprofloxacin-rifampin-ethambutol and the group receiving standard triple therapy.

Macrolides are the foundation of treatment for MAC-PD and the development of macrolide-resistant MAC-PD is associated with poor outcomes [12]. Macrolide therapy without ethambutol increases the risk of macrolide resistance [12]. Deviation from recommended therapy due to adverse events, and using macrolide monotherapy or macrolide with only a rifamycin or a fluoroquinolone are major causes for the development of macrolide resistance [13]. Among patients who received fluoroquinolone therapy in this study, 9/42 received a combination of macrolide-fluoroquinolone-rifamycin without ethambutol. Three of these patients had drug susceptibility testing $>6$ months after starting treatment and none developed macrolide resistance. However, given this very small number of patients, we cannot assess the likelihood of emergent macrolide resistance, a risk that bears consideration.

Since most of our patients (79\%) who received a fluoroquinolone also received a macrolide and ethambutol, our results provide more insight into the use of a fluoroquinolone in substitution for a rifamycin than for ethambutol. In light of recent evidence suggesting that macrolide-ethambutol may be equivalent to macrolide-ethambutol-rifamycin [14], the need for this substitution in the face of rifamycin avoidance requires further study.

Our study has several limitations. First, data collection was retrospective, so sputum cultures and computed tomography were performed according to clinical practice rather than strict schedule. Second, the small number of patients may have prevented us from detecting a significant difference between groups. Third, the decision to prescribe a fluoroquinolone was not standardised. Therefore, a prospective study is required for further assessment of the efficacy of fluoroquinolones for MAC-PD.

In conclusion, the present study demonstrated that three drug therapy with a fluoroquinolone, substituted most often for a rifamycin, resulted in similar treatment outcomes, but more adverse events, than standard triple therapy for MAC-PD.

Hadeel Khadawardi ${ }^{1,2,3}$, Theodore K. Marras ${ }^{1,2}$, Mahtab Mehrabi ${ }^{2}$ and Sarah K. Brode ${ }^{1,2,3}$

${ }^{1}$ Dept of Medicine, University of Toronto, Toronto, ON, Canada. ${ }^{2}$ Joint Division of Respirology, Dept of Medicine, University Health Network and Sinai Health System, Toronto, ON, Canada. ${ }^{3}$ West Park Healthcare Centre, Toronto, ON, Canada. 
Correspondence: Sarah K. Brode, Joint Division of Respirology, Dept of Medicine, University Health Network and Sinai Health System, Toronto Western Hospital, 399 Bathurst St. 7E-453, Toronto, ON M5T 2S8, Canada. E-mail: sarah. brode@uhn.ca

Received: 22 June 2019 | Accepted after revision: 29 Dec 2019

Conflict of interest: None declared.

\section{References}

1 Brode SK, Daley CL, Marras TK. The epidemiologic relationship between tuberculosis and non-tuberculous mycobacterial disease: a systematic review. Int J Tuberc Lung Dis 2014; 18: 1370-1377.

2 Griffith DE, Aksamit T, Brown-Elliott BA, et al. An official ATS/IDSA statement: diagnosis, treatment, and prevention of nontuberculous mycobacterial diseases. Am J Respir Crit Care Med 2007; 175: 367-416.

3 Kwak N, Park J, Kim E, et al. Treatment outcomes of Mycobacterium avium complex lung disease: a systematic review and meta-analysis. Clin Infect Dis 2017; 65: 1077-1084.

4 Diel R, Nienhaus A, Ringshausen FC, et al. Microbiologic outcome of interventions against Mycobacterium avium complex pulmonary disease: a systematic review. Chest 2018; 153: 888-921.

5 Field SK, Fisher D, Cowie RL. Mycobacterium avium complex pulmonary disease in patients without HIV infection. Chest 2004; 126: 566-581.

6 Griffith DE, Brown BA, Girard WM, et al. Azithromycin-containing regimens for treatment of Mycobacterium avium complex lung disease. Clin Infect Dis 2001; 32: 1547-1553.

7 van Ingen J, Wagner $\mathrm{D}$, Gallagher $\mathrm{J}$, et al. Poor adherence to management guidelines in nontuberculous mycobacterial pulmonary diseases. Eur Respir J 2017; 49: 1601855.

8 Sano C, Tatano Y, Shimizu T, et al. Comparative in vitro and in vivo antimicrobial activities of sitafloxacin, gatifloxacin and moxifloxacin against Mycobacterium avium. Int J Antimicrob Agents 2011; 37: 296-301.

9 Jenkins PA, Campbell IA, Banks J, et al. Clarithromycin $v s$ ciprofloxacin as adjuncts to rifampicin and ethambutol in treating opportunist mycobacterial lung diseases and an assessment of Mycobacterium vaccae immunotherapy. Thorax 2008; 63: 627-634.

10 Fujita M, Kajiki A, Tao Y, et al. The clinical efficacy and safety of a fluoroquinolone-containing regimen for pulmonary MAC disease. J Infect Chemother 2012; 18: 146-151.

11 Koh WJ, Hong G, Kim SY, et al. Treatment of refractory Mycobacterium avium complex lung disease with a moxifloxacin-containing regimen. Antimicrob Agents Chemother 2013; 57: 2281-2285.

12 Griffith DE, Brown-Elliott BA, Langsjoen B, et al. Clinical and molecular analysis of macrolide resistance in Mycobacterium avium complex lung disease. Am J Respir Crit Care Med 2006; 174: 928-934.

13 Morimoto K, Namkoong H, Hasegawa N, et al. Macrolide-resistant Mycobacterium avium complex lung disease: analysis of 102 consecutive cases. Ann Am Thorac Soc 2016; 13: 1904-1911.

14 Miwa S, Shirai M, Toyoshima M, et al. Efficacy of clarithromycin and ethambutol for Mycobacterium avium complex pulmonary disease. A preliminary study. Ann Am Thorac Soc 2014; 11: $23-29$. 